\title{
Prise en Charge de la Grossesse Extra-Utérine en Situation d'Urgence : Expérience d'un Hôpital Confessionnel en Zone Rurale au Togo
}

\author{
Biréga Koutora \\ Service de Chirurgie, \\ Hôpital de l'Ordre de Malte d'Elavagnon, Togo \\ Kodjo Abossisso Sakiye \\ Service des Urgences Chirurgicales, \\ CHU Sylvanus Olympio-Lomé, Togo \\ Déladem Yaovi Guinhouya \\ Service de Gynécologie-Obstétrique, \\ CHU Sylvanus Olympio-Lomé, Togo

\section{Mibirim Agbogawo} \\ Clinique Médico-Chirurgicale (Pavillon Militaire), \\ CHU Sylvanus Olympio-Lomé, Togo \\ Kokou Kouliwa Kanassoua \\ Département de Chirurgie et Spécialités chirurgicales, \\ Facultés des Sciences de la Santé, Université de Lomé, Togo
}

\section{Doi:10.19044/esj.2021.v17n14p237}

Submitted: 08 February 2021

Accepted: 31 March 2021

Published: 30 April 2021
Copyright 2021 Author(s)

Under Creative Commons BY-NC-ND

4.0 OPEN ACCESS

Cite As:

Koutora B., Sakiye K.A., Guinhouya D.Y., Agbogawo M. \& Kanassoua K.K. (2021). Prise en Charge de la Grossesse Extra-Utérine en Situation d'Urgence : Expérience d'un Hôpital Confessionnel en Zone Rurale au Togo. European Scientific Journal, ESJ, 17(14), 237.

https://doi.org/10.19044/esj.2021.v17n14p237

\section{Resume}

Introduction : La grossesse extra-utérine (GEU) est responsable d'une mortalité évitable. Le but de cette étude est de décrire la démarche diagnostique et thérapeutique de cette affection en zone rurale. Méthodologie : Il s'est agi d'une étude transversale descriptive menée à l'Hôpital de l'Ordre de Malte d'Elavagnon, et incluant les patientes prises en charge pour GEU du $1^{\text {er }}$ Décembre 2019 au 31 Décembre 2020. Résultats : Vingt-et-huit cas ont été enregistrés avec un âge moyen de 27,7 $\pm 5,4$ ans. Il 
s'agissait d'une GEU rompue dans tous les cas. Le diagnostic était essentiellement basé sur la clinique et le test de grossesse. Le traitement était chirurgical radical dans tous les cas. Le produit sanguin le plus utilisé était le sang total. Le pronostic était bon dans tous les cas. Conclusion : Le diagnostic de la GEU est essentiellement clinique et le traitement chirurgical en zone rurale.

Mots clés : Grossesse extra-utérine, Diagnostic, Traitement, Zone rurale

\title{
Care for Ectopic Pregnancy in Emergency Situation : Experience of a Confessional Hospital in Rural Zone in Togo
}

\author{
Biréga Koutora \\ Service de Chirurgie, \\ Hôpital de l'Ordre de Malte d'Elavagnon, Togo \\ Kodjo Abossisso Sakiye \\ Service des Urgences Chirurgicales, \\ CHU Sylvanus Olympio-Lomé, Togo \\ Déladem Yaovi Guinhouya \\ Service de Gynécologie-Obstétrique, \\ CHU Sylvanus Olympio-Lomé, Togo \\ Mibirim Agbogawo \\ Clinique Médico-Chirurgicale (Pavillon Militaire), \\ CHU Sylvanus Olympio-Lomé, Togo \\ Kokou Kouliwa Kanassoua \\ Département de Chirurgie et Spécialités chirurgicales, \\ Facultés des Sciences de la Santé, Université de Lomé, Togo

\begin{abstract}
Introduction : Ectopic pregnancy (EP) is responsible for preventable mortality. The aim of this study is to describe the diagnostic and therapeutic approach of this disease in rural zone. Methodology: Transversal study including patients treated for EP at Elavagnon « Ordre de Malte » Hospital, from $1^{\text {st }}$ December 2019 to $31^{\text {st }}$ December 2020. Results : Twenty-eight cases were registered with an average age of $27.7 \pm 5.4$ years old. The EP was ruptured in all cases. The diagnosis was mainly based on clinic and the pregnancy test. The treatment was radical surgery in all cases. The most used blood product was whole blood. The prognosis was good in all cases. Conclusion : EP diagnosis is mainly clinic and the treatment surgical in rural zone.
\end{abstract}

Keywords: Ectopic pregnancy, Diagnosis, Treatment, Rural zone 


\section{Introduction}

La grossesse extra-utérine (GEU) encore appelée grossesse ectopique est l'implantation et le développement de l'œuf fécondé en dehors de la cavité utérine. Son incidence est estimée à 1 à $3 \%$ des grossesses. Elle peut mettre en jeu le pronostic vital par le biais d'une rupture entrainant un hémopéritoine avec instabilité hémodynamique voire le décès par choc hypovolémique ; constituant à ce titre une urgence thérapeutique (Courbiere, 2009; Dohbit, 2010 ; Panelli, 2015). La GEU est responsable de 10\% de mortalité maternelle au premier trimestre de la grossesse (Kamga, 2017). La démarche diagnostique repose sur l'évaluation des facteurs de risque et le trépied clinique, biologique et échographique (Dessolle, 2002 ; Courbiere, 2009). L'arsenal thérapeutique a été enrichi du traitement médical et de l'abstention thérapeutique, qui, dans des indications spécifiques, sont des alternatives au traitement chirurgical. Ce dernier reste souvent la seule option thérapeutique dans les pays en développement où les patientes sont souvent reçues en situation d'urgence avec menace vitale. La coelioscopie s'est imposée comme la technique de référence pour la chirurgie de la grossesse tubaire ne laissant que peu de place à la laparotomie (Dessolle, 2002 ; Panelli, 2015). Cependant, son indisponibilité en Afrique rurale fait de la laparotomie, l'unique voie d'abord chirurgical. Cette étude a été initiée dans le but de décrire la démarche diagnostique et thérapeutique de la GEU en situation d'urgence dans un centre hospitalier confessionnel situé en zone rurale au Togo.

\section{Méthodologie}

Il s'est agi d'une étude transversale descriptive, menée à l'Hôpital de l'Ordre de Malte d'Elavagnon, du $1^{\text {er }}$ Décembre 2019 au 31 Décembre 2020 (13 mois). Ont été incluses, les patientes prises en charge dans ledit hôpital pour GEU. Le diagnostic était posé sur la base : de la clinique (douleurs pelviennes ou abdomino-pelviennes, métrorragies, et contexte de retard menstruel ou d'aménorrhée franche ; avec col utérin fermé et cri de Douglas) ; d'un test de grossesse positif (dosage qualitatif de $\beta H C G$ ); et de la culdocentèse. Le retentissement sur l'état hémodynamique était précisé. Une échographie pelvienne était réalisée au besoin. Le traitement consistait en une stabilisation de l'état hémodynamique par colloïdes et produits sanguins ; et un traitement chirurgical en urgence par laparotomie par abord de Pfannestiel. Les patientes sortaient de l'hôpital sous anti-anémique (Fer - Acide folique) et méthode contraceptive ; et étaient suivies en contrôles.

Les paramètres étudiés étaient: l'âge, la profession, les aspects diagnostiques et thérapeutiques, et le pronostic. Le traitement des données a été fait à partir du tableur Excel 2019. Les images ont été traitées sur le logiciel Paint. 


\section{Résultats}

\section{Aspects épidémiologiques}

Durant la période d'étude, 28 patientes ont été prises en charge pour GEU. Il s'agissait d'une GEU rompue dans tous les cas. L'âge moyen des patientes était de $27,7 \pm 5,4$ ans avec des extrêmes de 16 ans et 38 ans. Les patientes étaient majoritairement agricultrices $(42,8 \%)$. Les paramètres épidémiologiques sont récapitulés dans le tableau I.

Tableau I : Récapitulatif épidémiologique

\begin{tabular}{llrr}
\hline & & Effectif & $\%$ \\
\hline Tranche d'âge & $<25$ & 7 & 25,0 \\
& {$[25-35[$} & 17 & 60,7 \\
& $\geq 35$ & 4 & 14,3 \\
Profession & Agricultrices & 12 & \\
& Coiffeuses & 8 & 42,8 \\
& Couturières & 6 & 28,6 \\
& Accoucheuse & 1 & 21,4 \\
& Elève & 1 & 3,6 \\
& & & 3,6 \\
\hline
\end{tabular}

\section{Aspects diagnostiques}

La triade clinique associant douleurs pelviennes ou abdominopelviennes, métrorragies, et aménorrhée était présente dans 21 cas (75\%). Les métrorragies étaient absentes chez 7 patientes (25\%). On avait une syncope dans 3 cas $(10,7 \%)$. Vingt-et-six patientes $(92,9 \%)$ étaient en état de choc hypovolémique à l'admission; et $2(7,1 \%)$ en pré-choc. La culdocentèse était positive dans 23 cas $(82,1 \%)$. L'échographie était réalisée chez 7 patientes $(25 \%)$.

\section{Aspects thérapeutiques}

Pour la stabilisation de l'état hémodynamique, toutes les patientes avaient reçu un colloïde (Gélofusine ou Plasmion). La transfusion de produits sanguins était faite chez 19 patientes $(67,8 \%)$. Il s'agissait du sang total dans 17 cas $(60,7 \%)$, et du Concentré de Globules Rouges dans 2 cas $(7,1 \%)$. Le traitement chirurgical était réalisé en urgence. Il avait consisté en une laparotomie par abord de Pfannestiel dans tous les cas. A l'exploratoin chirurgicale, la grossesse tubaire était la plus retrouvée ( 24 cas / 85,7\%). La grossesse tubaire était ampullaire dans 23 cas et isthmique dans un cas. On avait 2 cas $(7,1 \%)$ de grossesse cornuale, un cas de grossesse ovarienne $(3,6 \%)$, et un cas de grossesse abdominale $(3,6 \%)$. Les figures $1,2,3$, et 4 sont des exemples d'images de GEU respectivement tubaire, cornuale, ovarienne, et abdominale retrouvées dans cette série. Le geste chirurgical effectué était fonction des constatations per-opératoires. La salpingectomie simple était le 
geste le plus réalisé $(57,1 \%)$. Le tableau 2 récapitule les gestes réalisés en fonction des lésions.

Tableau II : Récapitulatif des gestes réalisés en fonction des lésions

\begin{tabular}{lccc}
\hline & Effectif & Gestes réalisés & Effectif \\
\hline GEU tubaire & 24 & $\begin{array}{c}\text { Salpingectomie } \\
\text { Annexectomie }\end{array}$ & 16 \\
& & $\begin{array}{c}\text { Salpingectomie + Résection cornuale } \\
\text { Annexectomie + Résection épiplö̈que }\end{array}$ & 1 \\
GEU cornuale & 2 & Salpingectomie + Résection cornuale & 2 \\
GEU ovarienne & 1 & Annexectomie + Résection épiplöque & 1 \\
$\begin{array}{l}\text { GEU } \\
\text { abdominale }\end{array}$ & 1 & Anastomose du grêle + Appendicectomie & 1 \\
\hline
\end{tabular}

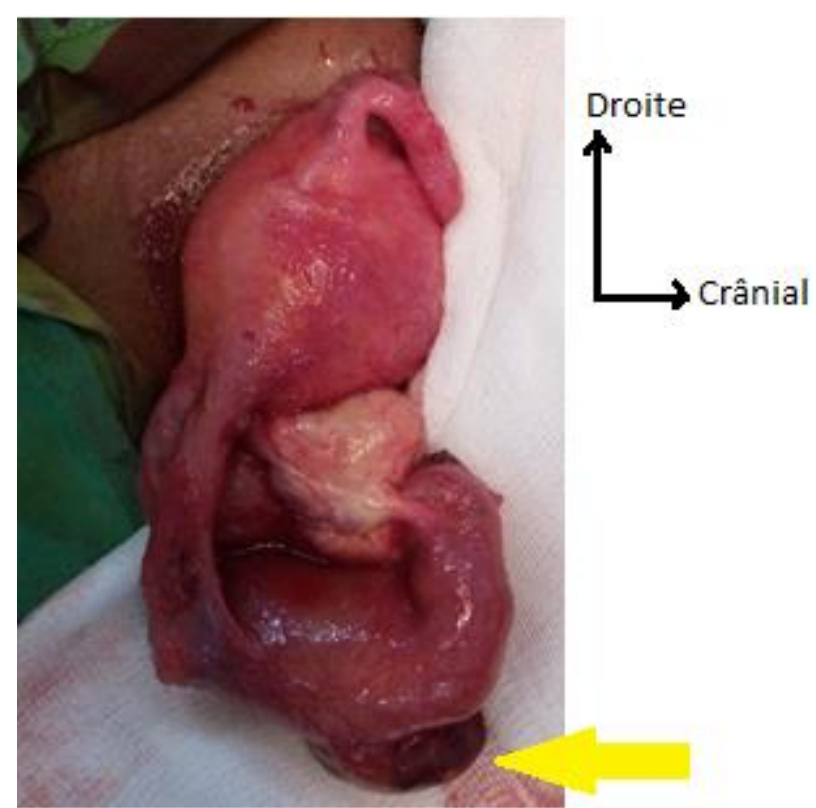

Figure 1 : GEU tubaire gauche (flèche) 


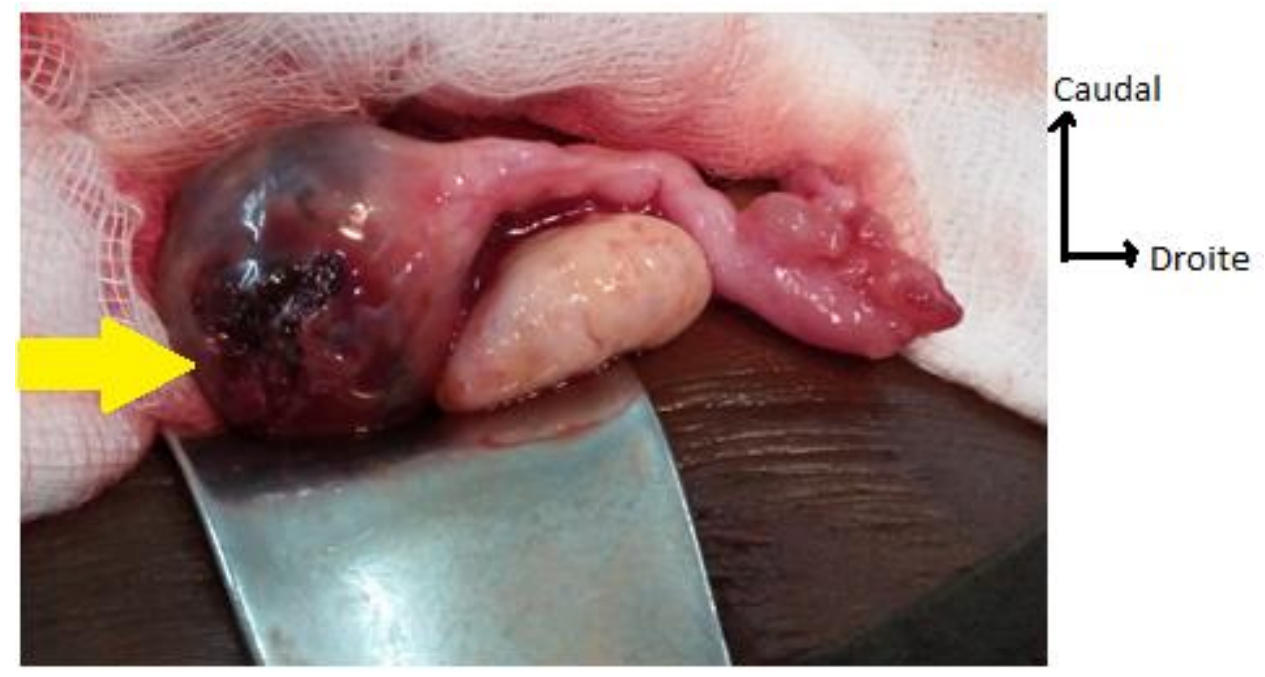

Figure 2 : GEU cornuale droite (flèche)

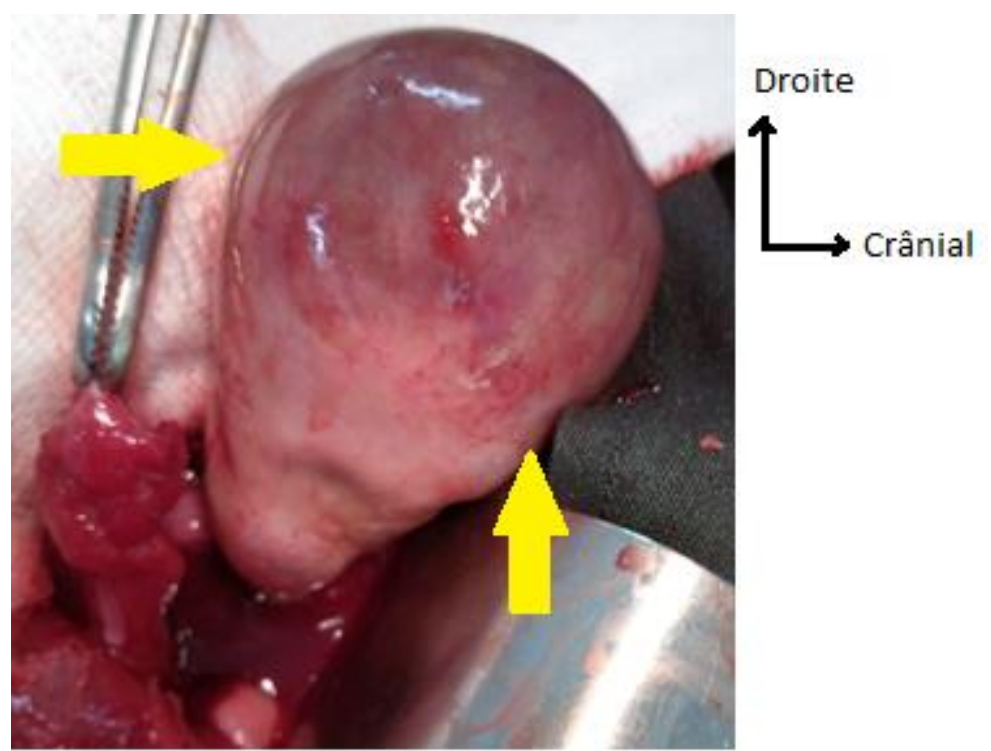

Figure 3 : GEU ovarienne droite (flèches) 


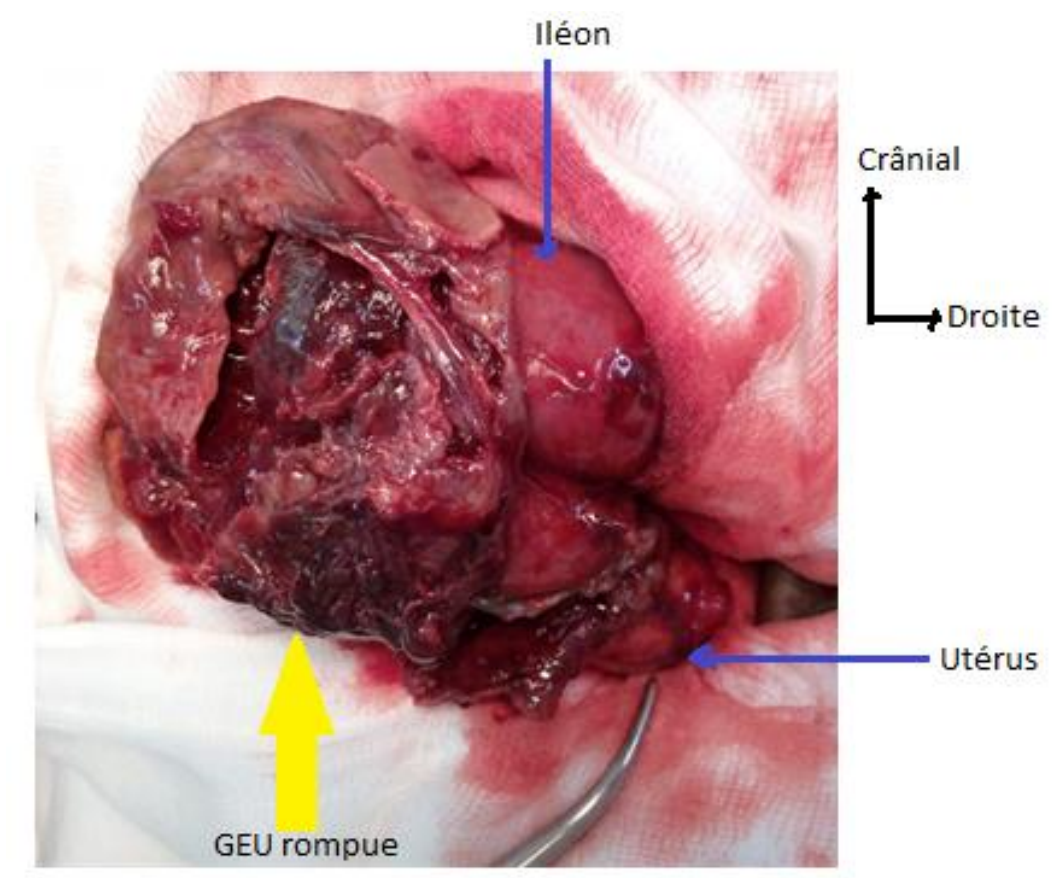

Figure 4 : GEU abdominale

\section{Aspects évolutifs}

Les suites opératoires étaient simples dans 27 cas $(96,4 \%)$. On avait un cas $(3,6 \%)$ d'infection du site opératoire. Il s'agissait d'une infection superficielle dont l'évolution était bonne sous les soins locaux. Le pronostic vital était bon dans tous les cas.

\section{Discussion}

La GEU est une urgence responsable d'une mortalité évitable de la femme jeune. Les progrès et la disponibilité des dosages hormonaux et l'échographie ont conduit à un dépistage plus précoce de cette pathologie avant la survenue des complications (Dessolle, 2002 ; Panelli, 2015). Dans cette série, la GEU était rompue dans tous les cas. Les difficultés d'accès aux soins de santé, les conditions de vie des populations mais aussi leur ignorance, pourraient expliquer le fait que la rupture de la GEU constitue souvent sa circonstance de découverte en Afrique rurale. L'âge moyen des patientes était de 27,7 ans avec une prédominance pour la tranche d'âge de 25 à 35 ans. Il s'agit en effet de la période de pleine activité sexuelle de la femme (Dohbit, 2010).

Sur le plan diagnostique, malgré la triade clinique caractéristique associant douleurs pelviennes, métrorragies et aménorrhées, il existe une variabilité symptomatique d'une patiente à l'autre (Kenfack, 2012 ; Kamga, 2017). Dans cette étude, la triade clinique était présente dans $75 \%$ des cas. On 
avait 3 cas de syncope. Toutes les patientes avaient un retentissement hémodynamique. Ainsi, toute manifestation clinique en rapport avec l'hémodynamie devrait faire penser à une GEU chez une femme sexuellement active. La culdocentèse était réalisée chez toutes les patientes de cette série. Ce geste simple, même s'il est supplanté par l'échographie est d'intérêt capital pour le diagnostic de la GEU rompue, permettant de faire économie du coût de l'échographie surtout chez les patientes en difficultés financières. Néanmoins, l'échographie est nécessaire afin de ne pas méconnaitre l'association d'une GEU et d'une grossesse intra-utérine réalisant une grossesse hétérotopique. Selon la topographie, la GEU tubaire était la plus fréquente dans cette série concordant avec les données de la littérature. La grossesse ovarienne et la grossesse abdominale n'étaient retrouvées que dans un cas chacune. Il s'agit en effet de situations rares (Dohbit, 2010; Ranaivoson, 2016 ; Guennoun, 2017 ; Sarr, 2018).

Le traitement de la GEU repose sur un arsenal thérapeutique enrichi de nos jours par le traitement médical et l'abstention thérapeutique. Il se pose souvent le problème de choix du traitement lorsque le diagnostic de la GEU est fait précocement avant la survenue des complications. On estime que près du tiers des GEU pourraient bénéficier soit d'un traitement médical, soit d'une abstention thérapeutique (Chapron, 2000 ; Dessolle, 2002). Cependant la GEU rompue impose souvent un traitement chirurgical. La stabilisation de l'état hémodynamique est faite à base de colloïdes, cristalloïdes, et produits sanguins. Le produit sanguin le plus utilisé dans cette série était le sang total. L'indisponibilité fréquente des produits sanguins dans ce milieu explique le recours au sang total grâce aux donneurs de sang prélevés sur place.

La GEU est responsable de près de $10 \%$ de mortalité maternelle au premier trimestre de la grossesse (Dessolle, 2002 ; Kamga, 2017). Dans cette série, la mortalité de la GEU était nulle, ceci du fait de la rapidité de la prise en charge grâce aux facilités offertes par l'hôpital notamment le système de paiement différé et l'assistance sociale aux malades.

\section{Conclusion}

La GEU est une urgence responsable d'une mortalité qui peut être évitée par une prise en charge rapide et adéquate. Son diagnostic repose essentiellement sur la clinique et le test de grossesse en zone rurale où la rupture constitue sa circonstance de découverte; et où l'accès aux soins est difficile. La présence des hôpitaux confessionnels en milieu rural à l'instar de l'Hôpital de l'Ordre de Malte d'Elavagnon est un atout pour les populations. L'assistance sociale aux malades et le système de paiement différé dans cette structure sanitaire permettent une meilleure gestion des urgences améliorant ainsi le pronostic vital. 


\section{Conflit d'intérêt : aucun}

\section{References :}

1. Chapron, C., Fernandez, H., \& Dubuisson, JB. (2000). Traitement de la grossesse extra-utérine en l'an 2000. J Gynécol Obstét Biol Reprod ; $29: 351-61$

2. Courbiere, R., \& Carcopino, X. (2009). Gynécologie Obstétrique, Ed Vernazobres-Grego

3. Dessolle, L., Detchev, R., \& Daraï, R. (2002). Chirurgie de la grossesse extra-utérine. Encycl Méd Chir, Techniques chirurgicales Gynécologie ; 41-530, 11p

4. Dohbit, JS., Foumane, P., Kaptche, MD., Mboudou, ET., Doumbe, M., \& Doh, AS. (2010). Grossesse extra-utérine à l'Hôpital Régional de Bafoussam: Aspects épidémiologiques, cliniques et thérapeutiques. Clin Mother Child Health ; 7(1) : 1189-93

5. Guennoun, A., Mamouni, N., Errarhay, S., Bouchikhi, C., \& Banani, A. (2017). La grossesse hétérotopique spontanée : à propos de deux cas. Pan Afr Med J ; 28 : 306 doi :10.11604/pamj.2017.28.306.13696

6. Kamga, DVT., Nana, NP., Fouelifack, FY., \& Fouedjio, JH. (2017). Contribution des avortements et des grossesses extra-utérines dans la mortalité maternelle dans trois hôpitaux universitaires de Yaoundé. Pan Afr Med J ; 27 : 248 doi :10.11604/pamj.2017.27.248.12942

7. Kenfack, B., Noubom, M., Bongoe, A., Tsatedem, FA., Ngono, M., Tsague GN., \& Mboudou, E. (2012). La grossesse extra-utérine dans une région semi-rurale en Afrique: Aspects épidémiologiques, cliniques et thérapeutiques à propos d'une série de 74 cas traités à l'Hôpital de District de Sangnelima au Sud-Cameroun. Pan Afr Med $\mathrm{J} ; 13: 71$

8. Panelli, DM., Phillips, CH., \& Brady, PC. (2015). Incidence, diagnosis and management of tubal and nontubal ectopic pregnancies : a review. Fertility Research and Practice ; 1 : 15 doi :10.1186/s40738-015-0008Z

9. Sarr, ISS., Faye, M., Faye, PM., Seck, M., Ka, O., \& Dieng, M. (2018). Occlusion intestinale aiguë révélant une grossesse abdominale : à propos d'un cas. Pan Afr Med J; 31: 155 doi :10.11604/pamj.2018.31.155.17187

10. Ranaivoson, HVR., Ranaivomanana, VF., Nomenjanahary, L., Andriamampionona, TF., \& Randrianjafisamindrakotroka, NS. (2016). Grossesse ovarienne : à propos de 3 cas et une revue de la $\begin{array}{llllll}\text { littérature. Pan Afr } & \text { Med J } & \text { 25: } & 128\end{array}$ doi :10.11604/pamj.2016.25.128.10834 